\title{
MedienPädagogik
}

Zeitschrift für Theorie und Praxis der Medienbildung

\section{Lehren lernen mit digitalen Medien}

\section{Technologiegestützte Praxisbezüge in der} Lehrpersonenbildung

Falk Scheidig

\section{Zusammenfassung}

Die individuelle Professionalisierung und der Erwerb einschlägiger Kompetenzen sind wesentliche Ziele der Lehrpersonenbildung. Die (mangelnde) Praxisorientierung von Lehramtsstudiengängen wird jedoch seit Langem kontrovers diskutiert. Während sich die Aufmerksamkeit vor allem auf Praktika (und deren Ausdehnung) richtet, erlaubt der Einsatz digitaler Technologien in der Hochschullehre wie etwa Onlineplattformen für kollaborative Videoannotation, Eүe-Tracking oder Virtual-Reality-Simulationen neue Formen der technologiegestützten Bezugnahme auf Praxis. Der Beitrag gibt einen Überblick über digitale Praxisbezüge, zeigt Beispiele und diskutiert deren Potenziale. 


\title{
Learning to teach with digital media. Technology-based references to professional practice in teacher education
}

\begin{abstract}
Professional development and the acquisition of relevant competences are main goals in pre-service teacher education. However, there is a long and controversial discourse about the (lack of) practical orientation of such study programs. While internships (especially their extension) receive a lot of attention, digital technologies in higher education such as collaborative video-annotation tools, eye-tracking systems or virtual reality simulations enable new forms of connections to professional practice. This article provides an overview of technology-based references to professional practice, presents examples and discusses their potential.
\end{abstract}

\section{Praxisbezüge in der Lehrpersonenbildung}

Das Herstellen von Praxisbezug - verstanden als direkte Bezugnahme auf berufliches Handeln in einem mit dem Studiengang korrespondierenden Feld - ist ein ebenso zentrales wie fortwährend Diskussionsanlass stiftendes Motiv von Lehramtsstudiengängen (zur Diskussion vgl. etwa jüngst Rothland 2020). «Eine Grundüberzeugung engagierter Lehrerbildung geht dahin, dass es nicht genug 〈Praxisbezug〉 geben könne, sondern immer nur $\mathrm{zu}$ wenig» (Oelkers 2000, 2). Es ist die Rede von einer «praxisbornierten Lehrerausbildung» (Hedtke 2019), von «Praxisparolen»(Wenzl, Wernet, und Kollmer 2018), vom «Wunsch nach mehr Praxis» (Makrinus 2013), vom «Mythos Praxis» (Rehfeldt et al. 2018; siehe auch Hascher 2011) bzw. der «Zauberformel Praxis» (Schrittesser et al. 2014).

Vielfach wird Praxisbezug verkürzend mit der Möglichkeit gleichgesetzt, in studienintegrierten Praktika in das Praxisfeld einzutauchen und Unterricht unmittelbar zu erleben und zu gestalten. Die Verlängerung, Anlage und Begleitung von Praxisphasen im Lehramtsstudium wird intensiv diskutiert (Arnold, Gröschner, und Hascher 2014; Artmann et al. 2018; Caruso 2019; Hesse und Lütgert 2020; Košinár, Gröschner, und Weyland 2019; Reintjes, Bellenberg, und im Brahm, 2018; Rheinländer und Scholl 
2020; Rothland und Biederbeck 2018; Ulrich und Gröschner 2020; Weyland und Wittmann 2015). Die Praxisphasen und -bezügen zugedachten Funktionen sind dabei ebenso breit gefächert wie die Umsetzung an den einzelnen Hochschulstandorten (vgl. z.B. Arnold et al. 2011; Bäuerlein und Fraefel 2016; Berndt, Häcker, und Leonhard 2017; Rothland und Boecker 2014; Weyland und Wittmann 2011; Winkel et al. 2020) und stehen in Abhängigkeit vom Zeitpunkt im Studienverlauf:

- in das Berufsfeld aus Lehrpersonenperspektive eintauchen, mit Bedingungen schulischen Handelns vertraut machen;

- den Berufswunsch, die Fächer- und Stufenwahl überprüfen;

- Überzeugungen und Vorstellungen aus der Schulzeit offenlegen und hinterfragen,

- Unterricht gestalten, Handeln einüben, unterrichtliche Kompetenz entwickeln;

- Wissen aus dem Studium mobilisieren, exemplifizieren und verstehen, Theorie und Praxis relationieren;

- Unterricht analysieren, reflektieren, beurteilen, Handlungsalternativen elaborieren;

- unterrichtliche Kompetenzen demonstrieren, dokumentieren, einer Beurteilung zuführen;

- individuelles Feedback zu Unterrichtshandeln, Mentoring und Coaching erhalten;

- Unterricht erforschen, Wissen generieren;

- die Zielorientierung adressieren, Motivation für Studium und Beruf bestärken.

Eine systematisierende Verdichtung nimmt Weyland (2010) vor. Sie unterscheidet drei Bezugssysteme der Lehrpersonenbildung und der Intention schulpraktischer Studien: erstens die Wissenschaft als Bezugssystem mit erkenntnisbezogener Perspektive und in Verbindung mit dem Ziel der Förderung der theoretischen Reflexionsfähigkeit und forschungsmethodischer Fähigkeiten, zweitens die Praxis als Bezugssystem mit handelndpragmatischer Perspektive sowie drittens die (angehende) Lehrperson in einer selbstreflexions- und entwicklungsbezogenen Perspektive. Insbesondere die Perspektiven des Wissenschafts- und Praxisbezugs können als 
divergierend wahrgenommen werden (z.B. Rothland 2020), besitzen aber auch Verbindungslinien (z.B. Tremp 2018) und sind in ihrer Dualität zu hinterfragen (Leonhard 2018).

Das Funktionsspektrum schulpraktischer Studienanteile ist insofern überlappend, als einige der oben aufgelisteten Funktionen quer zu anderen Funktionen liegen bzw. darin aufgehen, und soll deshalb hier aus einer Makroperspektive als heuristischer Entwurf (siehe auch Abschnitt 3) entlang von studentischen Tätigkeiten (die selbst eine Funktion von Praxisanteilen im Studium sind) strukturiert werden. Als studentische Tätigkeiten auf der Makroebene zur Kontaktnahme mit Schul- und Unterrichtspraxis werden drei Grundformen differenziert:

A. Das Beobachten und teilnehmende Erleben von Praxis (= in situ, passiv), insbesondere im Rahmen von Hospitationen.

B. Das Gestalten von Praxis (= in situ, aktiv), also die Übernahme von Verantwortung für Unterricht, dies umschliesst auch die Planung, das Erproben von didaktischen Settings, das Üben von Handlungspraktiken und das Demonstrieren von Unterrichtskompetenz.

C. Das situationsdistanzierte Analysieren von Praxis (= ex situ), hierzu zählen auch die retrospektive Reflexion von Praktika und die Beurteilung von Unterrichtssituationen.

Diese Makroformen sind keineswegs disjunkt, so bildet etwa die strukturierte teilnehmende Beobachtung mit Protokollbögen zu Analysezwecken ein Scharnier zwischen $(A)$ und $(C)$, Co-Teaching integriert (A) und (B) und die dem praktischen Handeln immanente Situationsdeutung zum Zwecke der Aufrechterhaltung situationsadäquater Handlungsfähigkeit verweist auf die Verbindung von (B) und (C). Gleichwohl handelt es sich hierbei um in der Lehrpersonenbildung tradierte und oftmals auch curricular gesondert ausgewiesene Praxiszugänge (z.B. als Hospitationspraktikum, Praxissemester und Praktikumsbegleitveranstaltung). Mit diesen drei Grundformen sind je spezifische Ziele intendiert, z.B. Handlungs- und Reflexionskompetenz zu entwickeln und unter Beweis zu stellen. Weitere Funktionen, wie sie oben aufgelistet wurden, liegen - auch in Abhängigkeit von der konkreten Konfiguration dieser Makroformen im Studiengang - quer zu den drei Grundformen und verbinden diese (siehe Tabelle 1). 
Jenseits immersiver Praxisbezüge im Rahmen konventioneller Praktika bestehen ebenfalls Optionen mittelbarer Praxisbezüge, z.B. die Analyse von Praxisartefakten, die (stellvertretende) Bearbeitung typischer Praxisprobleme, die Reflexion praktischer Erfahrungen, die Simulation von Praxisfacetten, der Dialog mit Personen aus der Praxis (Baer-Baldauf 2020; Scheidig 2017). Die Auseinandersetzung mit der flüchtigen, orts- und zeitgebundenen Schulpraxis erfolgte hierbei lange Zeit weitgehend sprachbzw. textvermittelt (mündliche Rede, Verschriftlichung wie Praktikumsberichte oder Praxisdokumente wie Unterrichtsplanungen), partiell auch auf Basis von audiovisuellen Unterrichtsmitschnitten.

\begin{tabular}{|l|c|c|c|}
\hline $\begin{array}{l}\text { Makroform des } \\
\text { Praxisbezugs }\end{array}$ & $\begin{array}{c}\text { Praxis beobachten } \\
\text { (z.B. Hospitations- } \\
\text { praktikum) }\end{array}$ & $\begin{array}{c}\text { Praxis gestalten } \\
\text { (z.B. Praxissemes- } \\
\text { ter) }\end{array}$ & $\begin{array}{c}\text { Praxis analysieren } \\
\text { (z.B. Praktikums- } \\
\text { begleitseminar) }\end{array}$ \\
\hline Situationsbezug & in situ, passiv & in situ, aktiv & ex situ \\
\hline $\begin{array}{l}\text { Querliegende } \\
\text { Funktionen }\end{array}$ & $\mathrm{X}$ & $\mathrm{X}$ & - \\
\hline $\begin{array}{l}\text { Eintauchen in das } \\
\text { Berufsfeld }\end{array}$ & $\mathrm{X}$ & $\mathrm{X}$ & - \\
\hline $\begin{array}{l}\text { Überprüfen der } \\
\text { Berufs- und Studi- } \\
\text { enwahl }\end{array}$ & $\mathrm{X}$ & - & $\mathrm{X}$ \\
\hline $\begin{array}{l}\text { Hinterfragen von } \\
\text { Vorstellungen aus } \\
\text { der Schulzeit }\end{array}$ & $\mathrm{X}$ & $\mathrm{X}$ & $\mathrm{X}$ \\
\hline $\begin{array}{l}\text { Relationieren von } \\
\text { Theorie und Praxis }\end{array}$ & $\mathrm{X}$ & $\mathrm{X}$ & $(\mathrm{X})$ \\
\hline $\begin{array}{l}\text { Motivieren für Stu- } \\
\text { dium und Beruf }\end{array}$ & - & $\mathrm{X}$ & $(\mathrm{X})$ \\
\hline $\begin{array}{l}\text { Erhalten von Feed- } \\
\text { back }\end{array}$ & & & \\
\hline
\end{tabular}

Tab. 1.: Strukturierung von Praxisbezügen im Lehramtsstudium.

Infolge verschiedener technologischer Entwicklungen, die zu Innovationssprüngen bei Hard- und Software, Kostenreduktion und nahezu flächendeckender Verbreitung von «mobile devices» führten, verzeichnen Möglichkeiten medienbasierter Praxisbezüge in den vergangenen Jahren 
eine substantielle Erweiterung. So werden an einigen Hochschulen etwa EPortfolios oder Virtual-Reality-Lernumgebungen in der Lehrpersonenbildung eingesetzt und hinsichtlich ihrer Wirkung erforscht. Als im Zuge der Corona-Pandemie Schulunterricht und Hochschullehre phasenweise flächendeckend nicht mehr in Präsenz stattfinden konnten und folglich auch Schulpraktika in Lehramtsstudiengängen kompensiert werden mussten, verhalf dies digitalen Formaten des Praxisbezugs wie der computerbasierten Nutzung von Unterrichtsvideos oder Schulsimulationen zu weiterem Auftrieb (Sasaki et al. 2020; Stringer Keefe 2020).

Gleichwohl existiert - trotz zahlreicher internationaler Beispiele und einer Verständigung über einzelne Zugänge wie Eye-Tracking oder Virtual Reality - bislang nur in Ansätzen und dies auch nur im englischsprachigen Raum ein übergreifender Diskurs, der verschiedene digitale Praxisbezüge als solche identifiziert und im Lichte des Praxisbezugs im Lehramtsstudium vernetzt (Herbst et al. 2016; Hixon und So 2009; Sweeney, Milewski, und Amidon 2018). Dies soll im Folgenden zum Anlass genommen werden, um im Rahmen dieses Beitrags das Feld digitaler Praxisbezüge skizzenhaft zu kartieren und mit ausgewählten, vorrangig dem deutschsprachigen Hochschulraum entstammenden Beispielen zu kolorieren (siehe Abschnitt 2). Hieran anknüpfend sollen die vorgestellten Formate in ordnender Absicht diskutiert werden (siehe Abschnitt 3), ehe der Beitrag mit weiterführenden Perspektiven schliesst (siehe Abschnitt 4). Damit verbindet sich das Ziel, in das Themenfeld einzuführen, es im deutschsprachigen Lehrerinnen- und Lehrerbildungsdiskurs zu verorten sowie ferner dazu anzuregen, die bisher weitgehend separat betrachteten Formate digitalen Praxisbezugs im Gesamtkontext des (technologiegestützten) Herstellens von Praxisbezügen zu diskutieren.

\section{Formate digitaler Praxisbezüge}

Digitale Formen der Bezugnahme auf Praxis sind zu unterscheiden von digitalen Formen des Handelns in der Praxis, etwa dem Einsatz von AudienceResponse-Systemen, Lern-Apps, Wikis, Lernplattformen oder Augmented Reality im Schulkontext. Der Fokus des vorliegenden Beitrags richtet sich auf erstgenanntes, auf technologiegestützte Zugänge zur Praxis im 
Rahmen des Lehramtsstudiums - im Sinne von Kontaktnahmen mit der Schule als Lern- bzw. Arbeitsort, dem Lehrberuf als Zieldimension des Studiums bzw. der angestrebten Tätigkeit des Unterrichts im Schulkontext. Als solche werden im Weiteren fünf Formate näher beleuchtet: Video Learning (Abschnitt 2.1), E-Portfolios (Abschnitt 2.2), Eye-Tracking (Abschnitt 2.3), Serious Games (Abschnitt 2.4) und Virtual Reality (Abschnitt 2.5). Die vorgenommene Kategorisierung dieser Formate digitalen Praxisbezugs ist weder trennscharf noch abschliessend, so lassen sich z.B. Serious Games und Virtual Reality als Subformen der Kategorie Simulation rubrizieren (Scheidig, im Erscheinen) oder Arten von Video Learning weiter differenzieren (Scheidig 2020a). Ebenso lassen sich der digitale Fernunterricht durch Studierende während der Corona-Pandemie (als Online-Praktika oder extracurriculare Hilfsleistungen im Bildungsbereich) als Mischform digitaler Bezugnahme auf und digitalen Handelns im Schulunterricht einordnen, gleiches gilt für Zweiwege-Videokonferenzen, bei denen Studierende via Bildschirm mit Schülerinnen und Schülern im Klassenraum interagieren (Sweeney, Milewski, und Amidon 2018).

Für die Kategorienbildung von Formaten digitalen Praxisbezugs wurde eine Recherche in internationalen Periodika und Proceedings zu Bildungsforschung und -technologie einbezogen. Diese erfolgte über das Portal LearnTechLib (www.learntechlib.org), einer Plattform, die u.a. Zugang zu 37 internationalen Journalen für Lehrpersonenbildung bietet; Suchbegriffe waren «practice based», «practical orientation», «professional practice», «practical experience», «field experience», «field placement», «practicum», «internship» und der Zeitraum die Jahre 2015 bis 2020. Anschliessend wurden anhand der unterschiedenen Formate über das Fachportal Pädagogik des DIPF (www.fachportal-paedagogik.de) deutschsprachige Beispiele gesucht; dies wurde punktuell durch weitere Literaturzugänge angereichert (u.a. teaching.org, Hochschulforum Digitalisierung, Monitor Lehrerbildung, Google Scholar). Diese Recherche legte offen, dass sich vor allem für den Einsatz von Video Learning und E-Portfolio im deutschsprachigen Raum dokumentierte Beispiele finden lassen - von denen nachfolgend nur wenige referenziert werden können - für Eye-Tracking, Serious Games und Virtual Reality hingegen nur vereinzelt. Die für diesen Beitrag vorgenommene Auswahl berücksichtigt dabei prioritär jene Beispiele, 
denen ein hohes Illustrationspotential zugeschrieben werden darf und zu denen idealiter auch Forschungsbefunde in publizierter Form vorliegen.

Die nachfolgende Zusammenstellung ist dabei nicht als Systematic Review (Cooper und Hedges 2009; Newman und Gough 2020) zur strukturierten und formatübergreifenden Darstellung des Forschungsstands in Bezug auf mehrere digitale Formate des Praxisbezugs angelegt. Es liegen jeweils bereits zum Teil sehr umfassende Reviews zu Video Learning (Blomberg et al. 2013; Gaudin und Chaliès 2015), (E-)Portfolios (Cramer und Feder 2018; Hofmann et al. 2016; Strudler und Wetzel 2011), Eye-Tracking (Beach und McConnel 2019) und Virtual Reality (Billingsley et al. 2019) im Kontext des Lehramtsstudiums vor. Bezüglich des Einsatzes von Serious Games in Lehr-/Lernkontexten liegen mehrere Reviews vor (Borges et al. 2014; Caponetto, Earp, und Ott 2014; Dichev und Dicheva 2017; Dicheva et al. 2015; Nah et al. 2014), obschon sich diese nicht spezifisch auf die Lehrpersonenbildung beziehen. In Ergänzung dieser Übersichtsarbeiten (in denen die nachfolgend genannten Beispiele notabene mehrheitlich keine Berücksichtigung erfahren) soll hier eine zunächst formatbezogene und anschliessend -verbindende thematische Einführung mit Anschlüssen an den deutschsprachigen Diskurs zur Lehrerpersonenbildung gegeben werden.

\subsection{Video Learning}

Bereits seit mehreren Jahrzehnten werden Unterrichtsvideos in der Lehrpersonenbildung genutzt, in den 1960er Jahren noch in aufwändigen «Unterrichtsmitschauanlagen» (Meyer und Aulinger 2019; Petko, Prasse, und Reusser 2014). Obgleich seitdem die (vorwiegend technischen) Hürden der Videoaufzeichnung und -präsentation deutlich gesunken und die didaktischen Möglichkeiten gestiegen sind, findet ein flächendeckender Einsatz bislang nicht statt. Unterrichtsvideografie fungiert als «(window) into teaching without the pressure of having to interact in the classroom situation» (Blomberg et al. 2013, 93) und speichert authentische, hoch verdichtete Unterrichtswirklichkeit als Videodatei, die wiederholt, mit verschiedenen Einstellungen, Foki und «theoretischen Brillen» betrachtet werden kann. Daneben ermöglicht die dabei verwendete Technik prinzipiell auch 
Live-Übertragungen aus der Praxis direkt ins Hochschulseminar (für ein Beispiel vgl. Bucheli 2018).

Unterrichtsvideos können in Lehrveranstaltungen, im Selbststudium, in «video clubs» (Sherin und van Es 2009; Tochon 1999) oder auch in Prüfungen verwendet werden (für eine Übersicht: Scheidig 2020a). Videoeinsatz erlaubt das Präsentieren und Analysieren von «best practice», «typical practice» oder «critical incidents》 (Friesen et al. 2020; Petko, Prasse, und Reusser 2014), das Profilieren einer multiperspektivischen, differenzierten und theoriegeleiteten Unterrichtswahrnehmung ("professional vision», Seidel und Stürmer 2014; Sherin und van Es 2009) und die fachlich begründete Elaboration von situationsbezogenen Handlungsoptionen (Gaudin und Chaliès 2015; Seidel, Blomberg, und Stürmer 2010; Sherin und van Es 2009).

Eine zentrale Ressource sind Plattformen mit bzw. für Unterrichtsvideos. Diese weisen unterschiedliche Funktionsumfänge auf, z.B. Suchfilter oder die Bereitstellung von ergänzendem Material wie Kontextualisierungshinweise, Stundenverlaufspläne, Analyseimpulse, Transkripte, Lehrpersonenkommentare und Expert*innenratings (für eine aktuelle Übersicht zu Videoportalen der Schul- und Unterrichtsforschung: www. fdz-bildung.de/videoportale). Ein Beispiel für die Konfiguration solcher Plattformen als Lernumgebung ist das Portal «UnterrichtOnline» der LMU München (Meyer und Aulinger 2019), das aus unterschiedlichen Perspektiven videografierte Unterrichtssequenzen (Fokus auf Lehrperson, Klasse, einzelne Schülerinnen und Schüler) mit verschiedenen Interaktionsoptionen verbindet (z.B. individuelle oder kollaborative Kommentierung der Videos und Nutzung eines anpassbaren Kategoriensystems). Die Onlineplattform «Multiview» der Universität Lüneburg stellt sogar Unterrichtsaufnahmen aus bis zu 16 verschiedenen Perspektiven zur Verfügung, dies u.a. in Verbindung mit Begleitmaterial aus sowie zu der Unterrichtsstunde (Claussen et al. 2020). Ein verwandtes Einsatzszenario ist die Einbettung von Unterrichtsvideos in ein hochschulisches Learning-Management-System (LMS), um z.B. der Bearbeitung von Aufgaben im Selbststudium einen Raum und Rahmen zu stiften (für ein Blended-Learning-Beispiel vgl. z.B. Bartel und Roth 2015). 
Sowohl einige Videoplattformen (z.B. edubreak, SWITCHcast annotate!) als auch LMS-Plug-ins (z.B. vShare) ermöglichen eine zeitstempelbasierte Markierung und Kommentierung konkreter Videostellen. Hieraus erwachsen Potentiale der (asynchronen) annotationsbasierten Videoanalyse im Web, die unter dem Terminus «Social Video Learning» diskutiert werden (Mayer-Frühwirth 2017; Vohle 2013). Dies bietet die Möglichkeit, studentische Eigenvideos - die in Praktika mit alltäglichen Digitalkameras, Camcordern oder neueren Smartphones und einem Stativ selbst oder gegenseitig aufgezeichnet werden können (Bäuerlein und Fraefel 2016) situierte Unterrichtserfahrungen detailliert zu analysieren, z.B. in Begleitveranstaltungen schulpraktischer Studien, in Blended-Learning-Szenarien oder im Wege online-basierten Peer-Lernens bzw. Peer-Feedbacks (vgl. z.B. König 2019).

In Abgrenzung zu mündlichen oder schriftlichen Praxisreflexionen ergeben sich ebenso für Dozierende und Praxismentor*innen neue Optionen der unverzerrten Einsicht in die Unterrichtsstunden angehender Lehrpersonen sowie eines hierauf gestützten zeitnahen Coachings und formativen Feedbacks schon während der Praxisphasen, um deren professionalisierungsfördernde Wirkung zu erhöhen (Gröschner, Klaß, und Winkler 2019). Beispiele für die sequentielle Verzahnung von videobasierter Selbstreflexion mit Online-Feedback durch Mitstudierende, Praktikumslehrpersonen und Dozierende in Praktika sind die LMS-integrierten Videoannotationstools V-Teach an der Universität Jena bzw. V-Reflect an der Universität Lüneburg (Kleinknecht und Gröschner 2016; Gröschner, Klaß, und Dehne 2018; Weber et al. 2018). Sequenzen von Eigenvideos können zudem in anderen Lehrveranstaltungen, z.B. der Fachdidaktiken, oder in studienbegleitenden E-Portfolios (siehe Abschnitt 2.2) referenziert werden und so im Sinne des vernetzten Wissensaufbaus, der Theorie-Praxis-Relationierung und der Kohärenzsteigerung die oftmals isolierten Praxiserfahrungen und -bezüge der Praktika mit anderen Lerngelegenheiten im Lehramtsstudium verbinden (Scheidig 2020b).

Neben diesen primär perzeptions-, reflexions- und feedbackorientierten Formaten online-basierter Videoarbeit lassen sich Unterrichtsvideos für Prüfungszwecke nutzen. Zum einen können Fremdvideos - alternativ: geskriptete (Piwowar et al. 2018) oder animierte Unterrichtsvideos (Herbst, 
Nachlieli, und Chazan 2011) - als Stimulus oder Bezugspunkt für Prüfungsaufgaben verwendet werden (vgl. z.B. Kersting 2008), u.a. für eine transferorientierte Wissensdarbietung («knowledge-based reasoning», Gaudin und Chaliès 2015; Seidel, Blomberg, und Stürmer 2010) in mündlichen Einzelund Gruppenprüfungen, in schriftlichen Seminar- bzw. Hausarbeiten oder eingebettet in computergestützte Tests (E-Assessment). Zum anderen ermöglichen studentische Eigenvideos eine performanzorientierte Dokumentation der Lehrkompetenz angehender Lehrpersonen (z.B. Baer et al. 2007), die u.a. im Hinblick auf Objektivität, Validität und Organisation Vorteile gegenüber konventionellen Lehrproben besitzen (Fraefel, Bäuerlein, und Barabasch 2018). Für die umfassende summative Kompetenzüberprüfung kann das Video in ein Dossier mit Stundenplanung, Unterrichtsmaterial und Reflexionsbericht eingebettet werden («Video-Portfolio», vgl. Bäuerlein und Fraefel 2016). Wie eine grossflächige, standardisierte und zugleich fach- und stufensensible Implementierung mit geschulten Ratern für vergleichbare Beurteilungen aussehen kann, exemplifiziert das edTPAProgramm (educational Teacher Performance Assessment), an dem aktuell 920 Lehrerbildungsprogramme aus 41 US-Bundesstaaten partizipieren (www.edtpa.com).

Zahlreiche internationale Forschungsarbeiten zu videobasierter Lehrpersonenbildung zeigen, dass Videos die fokussierte und interpretative Unterrichtswahrnehmung, die Reflexionstiefe sowie die Wissensaktivierung und -aneignung in der Lehrpersonenbildung fördern und die studentische Motivation und Zufriedenheit steigern; ob Videografie auch zur Weiterentwicklung bzw. Veränderung unterrichtlichen Handelns beiträgt, wird in Studien disparat beantwortet und ist empirisch schwer von anderen Einflussfaktoren zu isolieren (Gaudin und Chaliès 2015). Einschlägige Studien konstatieren ferner, dass die Erträge von den mit der Videoarbeit assoziierten Zielen und den verwendeten Videos abhängt: Eigenvideos werden als bedeutungsvoller wahrgenommen (Blomberg et al. 2013) und aktivieren eher das Vorwissen (Seidel und Thiel 2017), Fremdvideos befördern demgegenüber eher das Entwickeln von Handlungsalternativen (Kleinknecht und Schneider 2013). 


\section{$2.2 \quad$ E-Portfolio}

E-Portfolios stellen die digitale Weiterentwicklung von papierbasierten Portfolios dar; Gegenstand ist jeweils eine individuelle und strukturierte Kompilation von studienbezogenen Artefakten und deren Verbindung und Einordnung, um Lernprozesse und/oder Leistungen zu dokumentieren. Der Zugewinn von E-Portfolios gegenüber ihrem physischen Pendant besteht darin, dass diese erstens multimediale Zusammenstellungen erlauben (z.B. Integration von Ton- und Filmaufnahmen, animierten Grafiken, Präsentationen oder Hyperlinks), zweitens online geführt und somit jederzeit zugänglich, fortlaufend erweiterbar und kapazitär hinsichtlich Anzahl und Volumen der Artefakte kaum begrenzt sind und drittens die Möglichkeit bieten, das Portfolio - auch im Erstellungsprozess - mit Dozierenden, Mitstudierenden und weiteren Personen zu teilen, Rückmeldungen von ebendiesen empfangen und wieder in die Portfolioarbeit einfliessen lassen zu können.

E-Portfolios generieren einen Rahmen für die reflexive Auseinandersetzung mit Lernerfahrungen, können aber auch eine Funktionalisierung als formatives Entwicklungsinstrument oder summative Beurteilungsgrundlage erfahren (Baumgartner, Himpsl, und Zauchner 2009).

Es existieren zahlreiche Anwendungsbeispiele für hochschulische EPortfolios (Miller und Volk 2013), in der Lehrpersonenbildung finden sie bereits seit mehr als zehn Jahren Einsatz (z.B. Christen und Hofmann 2008; MacEntee und Garii 2010). Als online-geführte Sammlung erleichtern EPortfolios die Entkoppelung von einzelnen Lehrveranstaltungen, sie bieten neue Optionen multireferentieller Auseinandersetzung, insbesondere die Verknüpfung verschiedener Studienbereiche des Lehramtsstudiums im Sinne vernetzten Lernens und kohärenter Lehrpersonenbildung (Scheidig 2020b) sowie eine studienbegleitende oder sogar phasenüberschreitende, den Berufseintrittsprozess integrierende Dokumentation (Boos, Krämer, und Kricke 2016; Boulton 2014). An der Universität Kassel wird das E-Portfolio phasenübergreifend mit wechselnden Peer-Learning-Konstellationen genutzt (Busse und Bosse 2019): Das Feedback erfolgt zu Studienbeginn auf Basis bearbeiteter Aufgaben in Tandems untereinander sowie durch fortgeschrittene Studierende (E-Tutoren), im weiteren Studienverlauf dann reziprok in Tandems mit Referendarinnen und Referendaren auf der Basis 
von Texten sowie eigenen und fremden Unterrichtsvideos. In einer früheren Evaluation schätzten die Kasseler Lehramtsstudierenden vor allem den Charakter des E-Portfolios als Wissens- und Ideenspeicher sowie die Feedback- und Kollaborationsfunktionen (Karpa, Kempf, und Bosse 2013).

Insbesondere in schulischen Praxisphasen eignen sich E-Portfolios, um Erfahrungen multimedial zu dokumentieren, orts- und zeitungebunden zu teilen und Kommentare entgegenzunehmen. Neben E-Portfolio-Tools (z.B. Mahara) können auch praktikumsbegleitend geführte Blogs von Studierenden ein ähnliches Setup bieten (Totter 2019). Die webbasierte Führung von E-Portfolios eröffnet in Kombination mit der Omnipräsenz mobiler Endgeräte neue Optionen für Mobile Learning (Baran 2014; de Witt und Gloerfeld 2018). So erlaubt die an der PH Schwyz entwickelte App metapholio Studierenden, Praxissituationen und -eindrücke niedrigschwellig und ohne Zeitverlust als Text, Bild, Audio- oder Videomitschnitt festzuhalten, Dozierenden, Mentorinnen und Mentoren sowie Mitstudierenden zugänglich zu machen und diese Artefakte und hierauf bezogene Rückmeldungen später reflexiv zu verarbeiten (Petko et al. 2019). Scaffolding-Prompts in der App sollen zusätzlich die Reflexion stimulieren und die Theorie-Praxis-Relationierung fördern. In einer niederländischen Studie zum Einsatz einer ähnlichen App in Krankenhäusern reflektierten App-Nutzerinnen und -Nutzer häufiger und mit grösserem selbstberichteten Lernertrag als eine Vergleichsgruppe mit konventionellen Reflexionsnotizen (Könings et al. 2016). $\mathrm{Zu}$ beachten ist allerdings, dass Lehramtsstudierende das EPortfolio vor allem dann als lernförderlich erleben, wenn die Frequenz und Zeitpunkte des Feedbacks von Lehrer*innenbildner*innen sowie von Peers zeitlich angemessen sind und für sie selbst genügend Zeit zur reflexiven Verarbeitung besteht (Parker, Ndoye, und Ritzhaupt 2012).

Mit der Möglichkeit, Videos in E-Portfolios einzubetten, entfalten sich ferner Potentiale für Social Video Learning (siehe Abschnitt 2.1). Eine im Rahmen des Projekts PrepareCampus entwickelte Lernumgebung verbindet z.B. die online-kollaborative Videoannotation sequentiell mit der Unterrichtsreflexion: Studierende teilen und analysieren entlang von Aufgaben Eigenvideos, die anschliessend von Mitstudierenden und Mentorinnen und Mentoren kommentiert werden, ehe auf dieser Grundlage einzelne Sequenzen im persönlichen E-Portfolio reflektiert werden (Grubesic et al. 2018). 


\subsection{Eye-Tracking}

Mit Eye-Tracking können Situationen und Handlungen aus einer Akteursperspektive aufgezeichnet, videogestützt nachvollzogen und die Blickfolge, -foki und -fixationsdauer ausgewertet werden. Während Eye-Tracking zur Erkenntnisgewinnung in einigen Disziplinen und Anwendungsfeldern wie der Linguistik und Marktforschung seit langem eingesetzt wird (Duchowski 2002), finden sich in der empirischen Bildungsforschung wenige Beispiele (Jarodzka, Holmqvist, und Gruber 2017). Im Hinblick auf Anwendungsszenarien ist zwischen mobilen Eye-Tracking-Brillen (HeadMounted) und stationären Remote Eye-Trackern (Table-Mounted) zu differenzieren (Duchowski 2017):

Mit Eye-Tracking-Brillen lässt sich Unterricht aus der Perspektive der Lehrperson unter Einbezug der Blickbewegung aufzeichnen. Die Mitschnitte können sowohl zu videobasierten Unterrichtsanalysen als auch zur Thematisierung der visuellen Foki der Handelnden genutzt werden. Cortina et al. (2018) liessen angehende Lehrkräfte in den USA und Deutschland ebensolche Videos erstellen, anschliessend mündlich kommentieren (Thinking-Aloud-Methode) und konnten mittels strukturierter Inhaltsanalyse der Protokolle zeigen, dass sich die Reflexionen der Studierenden häufiger auf die Schülerinnen und Schüler richteten als auf die eigene Person, die beim Eye-Tracking nicht im Blickfeld ist. Sie sehen darin einen Vorteil gegenüber der Analyse von Unterrichtsvideos aus der Klassenraumperspektive, bei der die Fokussierung des Verhaltens der Lehrperson deutlich dominiert (Kleinknecht und Schneider 2013).

Neben der videobasierten Unterrichtsanalyse ermöglichen Eye-Tracking-Aufzeichnungen, die Unterrichtswahrnehmung der (angehenden) Lehrperson im komplexen Situationen zu einem späteren Zeitpunkt detailliert anhand der Blickbewegungen zu rekonstruieren und erörtern. Wie Kleiner, Rakoczi und Krimm (2012) anhand des Einsatzes im Sportunterricht demonstrieren, kann hierdurch die Aufmerksamkeitssteuerung optimiert und für Unterrichtsprozesse sensibilisiert werden, indem spezifische Momente und ausgewählte Unterrichtsaspekte mit Bezug zum Blickverhalten eine Thematisierung erfahren.

Remote Eye-Tracker werden demgegenüber an einer Bildausgabequelle positioniert, z.B. unterhalb des PC-Monitors, und zeichnen die 
Blickbewegung beim Betrachten des optischen Inputs (Video, Foto, Website, Software usw.) auf. In der Lehrpersonenbildung können auf diese Weise Unterrichtsvideos durch Studierende verfolgt und die Areale ihrer Aufmerksamkeit datengestützt ausgewertet werden, z.B. als Heatmap. Entsprechende Beispiele aus der Lehrpersonenbildung legen dar, dass erfahrene Lehrpersonen Unterricht umfassender und schneller erfassen als Novizinnen und Novizen, weil sich ihr Blick weniger lang auf einzelne Personen zentriert, sie verschiedene Unterrichtsprozesse parallel registrieren und sich ihr Fokus auf das Lernen der Schülerinnen und Schüler richtet (van den Bogert et al. 2014; Wolff, Jarodzka, und Boshuizen 2017). Ausgehend vom Befund, dass angehende Lehrpersonen relevante Situationen nicht missdeuten, sondern aufgrund des eingeschränkten Blickfokus gar nicht erst wahrnehmen (van den Bogert et al. 2014), kann dieses Setting als Grundlage für die Förderung einer «professional vision» jenseits situativen Handlungsdrucks im direkten Unterrichtsgeschehen genutzt werden, z.B. im Kontext der kompetenzorientierten Auseinandersetzung mit Classroom Management.

\subsection{Serious Games}

Das Potential von Computer- und Videospielen für Bildungszwecke darf als eingehend elaboriert gelten (Fromme und Petko 2008; Zheng und Gardner 2017). Sogenannte Serious Games stellen multimediale Informationsträger dar, die Lernpotentiale entfalten und mit spielerischen Elementen verknüpfen. Wouters et al. (2013) konstatieren in einer Metaanalyse, dass Serious Games - entgegen häufiger Annahmen - wenig motivationssteigernd wirken, aber positive Lerneffekte erzielen, vor allem wenn das Spielen durch andere Lehr-/Lernangebote und Mitlernende begleitet wird.

Verschiedene Simulationsspiele fokussieren schulisches Handeln und stehen für eine Nutzung in der Lehrpersonenbildung zur Verfügung (Bradley und Kendall 2014). Hierzu zählt u.a. simSchool, eine webbasierte Klassenraumsimulation mit Interaktionsaufgaben und Handlungsfeedback, deren Entwicklung am Anfang der 200oer Jahre vom Department of Education der USA gefördert wurde und in der aktuellen Version eine KIEngine (Künstliche Intelligenz) zur Verhaltens- und Emotionssteuerung 
der heterogen modellierten virtuellen Schülerinnen und Schüler integriert (Deale und Pastore 2014). Studien zum Einsatz von simSchool in der Lehrpersonenbildung weisen aus, dass Studierende die dargestellten $\mathrm{He}$ rausforderungen der virtuellen Lehrperson sowie die modellierten Personenprofile als realistisch einstufen (Badiee und Kaufman 2014) und sich das studentische Selbstwirksamkeitserleben und die berichtete Lehrkompetenzwahrnehmung durch das Spiel steigerten (Christensen et al. 2011; McPherson 2011). Auch Studien zu anderen Serious Games verweisen auf positive Effekte in der Lehrpersonenbildung, u.a. auf messbare Lernerträge hinsichtlich fachspezifischer Anforderungen (z.B. in Bezug auf Laborsicherheit im Chemieunterricht, vgl. Annetta et al. 2014) oder der Kooperation bei professionellen Herausforderungen des Lehrberufs (Hummel et al. 2013).

Ein Beispiel aus dem deutschen Raum ist das an der Universität $\mathrm{Pa}$ derborn entwickelte und in ein Blended-Learning-Konzept eingebettete Adventure-Spiel GEProS (Game- und e-learningbasierte, problemorientierte und selbstgesteuerte Lernumgebung). Thematisch im Zentrum steht die schulische Lerndiagnostik: Studierende sollen diagnostisches Wissen vertiefen und problembezogen anwenden, indem sie systematisch Fälle bearbeiten und dabei Beobachtungen und bereitgestellte Informationen gezielt als Ressource nutzen. Durch integriertes Lernmaterial und Feedback soll die Software die Reflexion und das Lernen der Studierenden anregen (Praetorius et al. 2016). Mittels einer Variation der gegebenen Informationen zu den Fällen (z.B. Zeugnisse, Klassenbucheinträge, Elterngespräche) lässt sich offenlegen und thematisieren, wie und welche Informationen auf diagnostische Prozesse der angehenden Lehrpersonen Einfluss nehmen (Klingsieck et al. 2016).

\subsection{Virtual Reality}

Computergestützte Simulationen wie Virtual Reality (VR) besitzen viele Gemeinsamkeiten mit Computer- und Videospielen. Im Unterschied zu Serious Games mit spielerischem und erkundendem, zuweilen auch unterhaltungsorientiertem und kompetitivem Charakter zielen pädagogische Simulationen stärker auf eine authentische Nachbildung einer real 
existierenden Praxis, um ein wirklichkeitsnahes Handeln (Immersion) zu ermöglichen (Imlig-Iten und Petko 2018; Kerres 2018, 12). Simulationen intendieren eine fokussierte Übernahme von Rollen und Verantwortung und akzentuieren die Anwendung und das Training relevanter Kompetenzen stärker als Spiele. Für die Lehrpersonenbildung sind sie u.a. relevant, weil sich das Handeln in spezifischen kritischen Situationen in Praktika aus ethischen und strukturellen Gründen nicht planvoll üben lässt.

Innovative Beispiele für Simulationen in der Lehrpersonenbildung stellen virtuelle Umgebungen dar, etwa das "VR-Klassenzimmer», mit dem Studierende an der Universität Potsdam Kompetenzen des Classroom Managements trainieren. Über ein VR-Headset (Head-Mounted Display, HMD) tauchen die Studierenden in eine dreidimensional visualisierte Unterrichtssequenz ein und steuern die Bewegungen und die Position des virtuellen Lehrpersonenavatars mit Controllern. Während eine angehende Lehrperson in der immersiven Lernumgebung in einem MicroteachingSetting unter Anwendung zuvor erarbeiteten Wissens zum Classroom Management agiert, kann das Verhalten der Schülerinnen und Schüler (Formen von Störungen oder Mitarbeit) im virtuellen Klassenraum durch Dozierende extern gesteuert und von den Mitstudierenden - z.B. strukturiert durch Analyseaufgaben - über einen Monitor oder Projektor live mitverfolgt werden (Wiepke et al. 2019). Auf dieser Basis können anschliessend die Handlungen im virtuellen Raum, diesbezügliche Interpretationen, Handlungsalternativen und relevantes Professionswissen diskursiv erschlossen werden. In einer Begleitstudie wird ein substantieller Zuwachs der wahrgenommenen Handlungskompetenz durch das virtuelle Training konstatiert und ebendieses seitens der Studierenden als bedeutsam eingestuft (ebd.).

Ein vergleichbar konfiguriertes Virtual-Reality-Projekt namens «Breaking Bad Behaviors» existiert auch an der Universität Würzburg (Lugrin et al. 2016). Basierend auf einem Prä-Post-Test zur Erfassung der Classroom-Management-Kompetenzen von Studierenden vor und nach dem Besuch einer betreffenden Lehrveranstaltung berichten Lugrin et al. (2018), dass der Kompetenzzuwachs bei Studierenden in der virtuellen Umgebung signifikant grösser ausfiel als bei einer Vergleichsgruppe, die mit Unterrichtsvideos arbeitete. In einem verwandten Projekt wird eine 
virtuelle Multi-User-Umgebung entwickelt, also ein geteilter VR-Raum, der mehrere studentische Avatare zulässt und ein vernetztes Agieren im virtuellen Unterricht erlaubt («Social Virtual Reality», Latoschik et al. 2019).

Virtual-Reality-Umgebungen für Lehrpersonen könnten künftig in verschiedener Hinsicht weiterentwickelt werden: Unterrichtsmitschnitte, die als 360-Grad-Videos (Spherical Video) aufgenommen werden, könnten eine Grundlage für immersive Simulationen bilden, die nicht auf künstlichen Computeranimationen fussen, sondern auf echtem Bildmaterial. Ausserdem könnten die Potentiale des Eye-Trackings auch beim virtuellen Unterrichten genutzt werden, wenn VR-Brillen (z.B. HTC Vive Pro Eye) nicht nur die virtuelle Umgebung darstellen, sondern auch das Blickverhalten ihrer Träger erfassen - wohin blicken Studierende wann und wie lange beim virtuellen Training des Umgangs mit Unterrichtsstörungen? Zudem wäre es möglich - in Anlehnung an das Beispiel simSchool - das Verhalten virtueller Schülerinnen und Schüler mittels Machine-LearningAlgorithmen realitätsnaher zu steuern. Dies liesse sich u.a. nutzen, um die Adaptivität der Simulation zu erhöhen. Für eine adaptive Simulationssteuerung könnten des Weiteren Körpersensoren einbezogen werden, z.B. für eine Anforderungsniveauregulierung anhand Stress-indizierender physiologischer Statusdaten wie der Herzfrequenz, die mit Wearables am Arm der Nutzenden gemessen und über eine Schnittstelle an die VR-Software übermittelt werden (Acerbi et al. 2017; für ein Beispiel aus dem Bereich der Rettungskräfteschulung vgl. Fortenbacher et al. 2017).

\section{Einordnung und Systematisierung}

Die im Vorangegangenen vorgestellten digitalen Praxisbezüge besitzen unterschiedliche Funktionalität und variieren hinsichtlich des Innovationscharakters: Mit Eye-Tracking, Serious Games und Virtual Reality eröffnen sich für angehende Lehrpersonen neuartige Zugänge zu Schule und Unterricht, die bislang wenig Verbreitung in der deutschsprachigen Lehrpersonenbildung fanden und bestehende Formen der Bezugnahme auf Praxis erweitern (können). Im Vergleich hierzu dürfen Unterrichtsvideos und Portfolios eher als bewährte Medien der Lehrpersonenbildung eingestuft werden, für deren Einsatz sich jedoch innovative Weiterentwicklungen 
infolge von Digitalisierungsprozessen ergeben (z.B. erweitertes Funktionsspektrum, räumlich und zeitlich flexible Bearbeitung, Annotation durch Peers und Dozierende).

Die fünf Formate liegen quer zu den drei Bezugssystemen der Lehrpersonenbildung Wissenschaft, Praxis und Person (Weyland 2010) und entfalten ihre Funktionalität im Hinblick auf zwei verschiedene Bezugspraxen: Es ist zu unterscheiden zwischen digitalen Praxisbezügen basierend einerseits auf einer realen Unterrichtspraxis, die z.B. videografiert wird, und andererseits basierend auf Abbildern der Praxis, die auf einem künstlichem Modell beruhen (Hixon und So 2009) und aus Studierendenperspektive als «Virtual Field Experiences》 gefasst werden (Sweeney, Milewski, und Amidon 2018). Beides sind Formen der Repräsentation, die medial transportiert werden, und Mischformen sind möglich, z.B. geskriptete Unterrichtsvideos, ebenso weitere Differenzierungen, z.B. können EPortfolio-Elemente sowohl «records of practice» (z.B. Video-, Audioaufnahmen, Fotos) als auch «transformations of practice» (z.B. Transkriptionen und Interpretationen) umfassen (Herbst et al. 2016).

Mit Blick auf diese Unterscheidung zwischen realen und simulierten Referenzpraxen sowie der eingangs vorgenommenen Strukturierung des Funktionsspektrums berufspraktischer Studienelemente (Tabelle 1) ergeben sich für die fünf hier vorgestellten Formate verschiedene Funktionsbereiche (Tabelle 2). Mit Videos, E-Portfiolios und Eye-Tracking kann in je spezifischer Weise eine reale Unterrichtspraxis erfasst und gespeichert, anderen präsentiert und auf dieser Basis u.a. erschlossen und beurteilt werden. Serious Games und Virtual-Reality-Umgebungen basieren demgegenüber auf einer simulierten Praxis und erlauben ein Probehandeln zur Exploration, Anwendung oder Vertiefung professioneller Kompetenzen. Alle Formen digitaler Praxisbezüge verbindet, dass ihr prozessieren eine technologiebasierte Mediennutzungspraxis mit Unterrichtsbezug generiert (z.B. das Agieren im virtuellen Klassenzimmer, die Relationierung von Videos und Fachwissen), die als studentische Lernhandlungen wiederum als Beitrag zur Professionalitätsentwicklung kriterial analysiert und perspektivoffen reflektiert werden können. Unterrichtsmitschnitte (inklusive mit Eye-Tracking-Technologie aufgezeichnete Videos) und E-Portfolios ermöglichen zudem die Analyse und Reflexion der in ihnen dokumentierten realen Unterrichtspraxis der Studierenden. 


\begin{tabular}{|l|c|c|c|}
\hline Verhältnis zur & $\begin{array}{c}\text { Praxis erfassen, } \\
\text { präsentieren, beur- } \\
\text { teilen }\end{array}$ & $\begin{array}{c}\text { Praxis simulieren, } \\
\text { gestalten, } \\
\text { üben }\end{array}$ & $\begin{array}{c}\text { Praxis analysieren, } \\
\text { reflektieren }\end{array}$ \\
\hline Grundlage & $\begin{array}{c}\text { reale Unterrichts- } \\
\text { praxis (eigen, } \\
\text { fremd) }\end{array}$ & $\begin{array}{c}\text { simulierte Unter- } \\
\text { richtspraxis (vir- } \\
\text { tuell) }\end{array}$ & $\begin{array}{c}\text { studentische digita- } \\
\text { le Praxisbezugnah- } \\
\text { me (medial) }\end{array}$ \\
\hline Medium & $\mathrm{X}$ & - & $\mathrm{X}$ \\
\hline Video Learning & $\mathrm{X}$ & - & $\mathrm{X}$ \\
\hline E-Portfolio & $\mathrm{X}$ & - & $\mathrm{X}$ \\
\hline Eye-Tracking & - & $\mathrm{X}$ & $\mathrm{X}$ \\
\hline Serious Games & - & $\mathrm{X}$ & $\mathrm{X}$ \\
\hline Virtual Reality & & & \\
\hline
\end{tabular}

Tab. 2.: Formen und Funktionen digitaler Praxiszugänge.

Während mit E-Portfolios gemeinhin eigene Unterrichtserfahrungen verarbeitet werden, kann Video Learning sowohl praxisbegleitend auf $\mathrm{Ba}$ sis studentischer Videos als auch entkoppelt von Praxisphasen mit Fremdvideos stattfinden. Serious Games und Virtual-Reality-Simulationen stehen in keinem direkten Zusammenhang mit Praktika. Unterrichtsvideografie, mobiles Eye-Tracking sowie E-Portfolios mit medial fixierten Praxismomenten sind insofern praxisinvasiv, als sie im schulischen Klassenzimmer Sichtbarkeit erlangen und potentiell Einfluss auf Beteiligte nehmen, woraus Anforderungen erwachsenen u.a. im Hinblick auf Planung und Organisation sowie Information, Einverständnis und Datenschutz (Sonnleitner et al. 2018).

Digitale Praxisbezüge sind prinzipiell für verschiedene (Meta-)Konzepte der Lehrpersonenbildung anschlussfähig, z.B. Kompetenzorientierung, Forschendes Lernen, Problembasiertes Lernen, rekonstruktive Fallarbeit. Auf übergeordneter Ebene verbinden sich mit ihnen generische hochschuldidaktische Ziele: Studierende motivieren, ihr Vorwissen aktivieren, dialogische Phasen anregen, Raum und Anlass für kooperative Lernformen stiften, abwechslungsreiche und innovative Lehrformate kreieren, Studierende mit digitalen Medien in Kontakt bringen (Hixon und So 2009). Wenngleich diese den Formaten digitalen Praxisbezugs zugeschriebenen Erträge nicht allein deren Einsatz legitimieren, dürfen sie - so sie eintreten - als erwünschte Begleiteffekte verstanden werden. Die Erhebungen 
zu den referierten Anwendungsbeispielen fundieren diese Erwartungen mehrheitlich in positiver Hinsicht, obschon Bedarf an weiterer Forschung besteht. Über die hochschuldidaktische Facette dieser Formate hinaus erwachsen Potentiale für die Forschung zur Lehrpersonenbildung, denn die Nutzung digitaler Formate des Praxisbezugs generiert Daten, die - unter Wahrung des Datenschutzes und Beachtung ethischer Dimensionen der Datennutzung (Scheidig und Holmeier, im Erscheinen) - LearningAnalytics-Zugänge eröffnet, z.B. die Auswertung studentischer Texterzeugnisse (Videoannotationen, Portfolioreflexionen) oder automatisiert erfasster Daten (Logdaten von Serious Games, Eye-Tracking-Daten) zur Gewinnung von Erkenntnissen über Prozesse der Kompetenzentwicklung, Wissensaneignung und -verwendung sowie die Nutzung von Lerngelegenheiten im Lehramtsstudium.

Weitere Funktionen und Subfunktionen digitaler Praxisbezüge lassen sich ebenso elaborieren wie Mischformen und Kombinationen der hier vorgestellten Zugänge. Die erwähnten Beispiele illustrieren, dass technologiegestützte Formen der Bezugnahme auf Schul- und Unterrichtspraxis mit verschiedenen Arbeits- und Sozialformen einhergehen können. Ebenso wenig wie digitale Praxisbezüge Praxisphasen substituieren, treten sie in Konkurrenz zu Präsenzlehrveranstaltungen an der Hochschule. Vielmehr mündet ihr Einsatz in eine Verschmelzung digitaler und «analoger» Lehr-/ Lernarrangements (Schön, Ebner, und Schön 2016), da eine inhaltliche und organisatorische Einbettung in Lehrveranstaltungen anempfohlen, wenn nicht gar zwingend erforderlich ist. Dies gilt auch für Serious Games, für die eine Einführung und Unterstützung der Lernenden angemahnt wird (Badiee und Kaufman 2014; Wouters et al. 2013). Nimmt man die Differenzierung hochschuldidaktischer Digitalisierungskonzepte von Bachmann et al. (2002) als Folie zur Einordnung, so vollzieht sich mit digitalen Praxisbezügen primär eine mediale Anreicherung der Lehrveranstaltungen, partiell auch eine Integration von Online- und (bzw. in) Präsenzphasen. Ein Virtualisierungskonzept, das Lernprozesse vollständig in den virtuellen Raum verlagert, liegt hingegen nicht vor. Der Charakter der vorgestellten Zugänge lässt sich eher als hybrid kennzeichnen, und zwar in doppelter Hinsicht - es verschränken sich konventionelle und digitale Lehr- und Lernformen an der Schnittstelle von Praxis und Studium. Die im Kontext 
verlängerter Praktika vernehmbaren Voten, dass - jenseits der zeitlichen Quantität der Erkundung, Erprobung und Reflexion praktischen Handelns in der Schule - der fachlichen Begleitung der Praxiserfahrungen besondere Bedeutung beizumessen ist und passende hochschuldidaktische Rahmungen zu konfigurieren sind (Fromm und Strobel-Eisele 2020; Hesse und Lütgert 2020; Reintjes, Bellenberg, und im Brahm 2018), dürfte für digitale Praxisbezüge im Studium ungemindert gelten, stellen sie doch ebenfalls berufsbezogene Lerngelegenheiten u.a. mit Potential für die Aktivierung und Relationierung wissenschaftlicher Wissensbestände dar.

\section{An- und abschliessende Perspektiven}

Mit dem Einsatz digitaler Praxisbezüge dürften sich nicht nur die ihnen zugesprochenen Potentiale in der Lehrpersonenbildung entfalten, sondern auch damit verbundene Limitationen, Herausforderungen und Entwicklungsperspektiven an Kontur gewinnen. «Much of the work currently being done with technology environments in teacher education is exploratory» (Herbst et al. 2016, 97). Korrespondierende und mit der Implementation zu verknüpfende Fragen betreffen neben hochschuldidaktischen, technischen und organisatorischen Aspekten etwa den Umgang mit Domänenspezifika (z.B. des Kunst-, Musik-, Sport-, Sprachen- oder Informatikunterrichts) oder den Datenschutz. Es schliessen sich weitere Fragen mit Forschungsreferenz an: Welche Lehr- und Lernformen evozieren digitale Praxisbezüge im Lehramtsstudium? Welche Effekte treten ein, z.B. im Hinblick auf Kompetenzentwicklung, Verarbeitungstiefe, Reflexionsfoki? Verfestigen sich durch technologiegestützte Praxiszugänge eindimensionale Bilder von Praxis, beispielsweise durch narrative Fälle - oder fördern gerade medial vermittelte und insofern situationsdistanzierte Praxiseindrücke ein Lösen vom Bestehenden und das Denken in Alternativen in einem geschützten Raum (Hixon und So 2009)? Lässt sich mittels medialer Repräsentation ein Nachvollzug komplexer Unterrichtssituationen und ein Authentizitätserleben herstellen, z.B. situativer Handlungsdruck näherungsweise nachempfinden, oder erfolgt eine Zentrierung auf die sichtbare Oberflächenstruktur von Unterricht? 
Zugleich schliessen die hier vorgestellten Szenarien an die Diskussion um die Digitalisierung von Schule und Lehrpersonenbildung an, in der Bedarfe und Herausforderungen eine besondere Betonung erfahren (Cress et al. 2018; KMK 2017; Petko, Döbeli Honegger, und Prasse 2018; van Ackeren et al. 2019). Dies findet seine Entsprechung auch in der Befundlage, denn Studien attestieren Lehrpersonen ebenso wie Lehramtsstudiengängen ein Digitalisierungsdefizit (so u.a. Drossel et al. 2019; Schmid et al. 2017), und insbesondere in schulpraktischen Studienanteilen wird digitalen Medien bislang wenig Aufmerksamkeit geschenkt (Monitor Lehrerbildung 2018). In diesem Kontext verbindet sich mit Formaten digitaler Bezugnahmen auf Unterrichts- und Schulpraxis nicht nur die Erwartung einer Steigerung des akademischen Praxisbezugs und der Förderung von Professionalisierungsprozessen angehender Lehrpersonen, sondern auch die Perspektive, dass der Kontakt mit digitalen Medien und die reflexive Durchdringung entsprechender Erfahrungen sowohl aufseiten der Studierenden als auch der Dozierenden medienpädagogisch wünschenswerte Erträge erzielt.

\section{Literatur}

Acerbi, Giorgia, Erika Rovini, Stefano Betti, Antonio Tirri, Judit Flóra Rónai, Antonella Sirianni, Jacopo Agrimi, Lorenzo Eusebi, und Filippo Cavallo. 2017. «A Wearable System for Stress Detection Through Physiological Data Analysis.》In Ambient Assisted Living. ForItAAL 2016. Lecture Notes in Electrical Engineering, vol 426, herausgegeben von Filippo Cavallo, Vincenzo Marletta, Andrea Monteriù, und Pietro Siciliano, 31-50. Cham: Springer. https://doi. org/10.1007/978-3-319-54283-6_3.

Annetta, Leonard, Richard Lamb, James Minogue, Elizabeth Folta, Shawn Holmes, David Vallett, und Rebecca Cheng. 2014. «Safe science classrooms: Teacher training through serious educational games.» Information Sciences 264: 61-74. https://doi.org/10.1016/j.ins.2013.10.028.

Arnold, Karl-Heinz, Alexander Gröschner, und Tina Hascher, Hrsg. 2014. Schulpraktika in der Lehrerbildung. Theoretische Grundlagen, Konzeptionen, Prozesse und Effekte. Münster: Waxmann.

Arnold, Karl-Heinz, Tina Hascher, Roland Messner, Alois Niggli, Jean-Luc Patry, und Sibylle Rahm, Hrsg. 2011. Empowerment durch Schulpraktika. Perspektiven wechseln in der Lehrerbildung. Bad Heilbrunn: Klinkhardt.

Artmann, Michaela, Marie Berendonck, Petra Herzmann, und Anke B. Liegmann, Hrsg. 2018. Professionalisierung in Praxisphasen der Lehrerbildung. Qualitative Forschung aus Bildungswissenschaft und Fachdidaktik. Bad Heilbrunn: Verlag Julius Klinkhardt. 
Bachmann, Gudrun, Martina Dittler, Thomas Lehmann, Dieter Glatz, und Fritz Rösel. 2002. «Das Internetportal «Learn Tec Net» der Universität Basel.»In Campus 2002, herausgegeben von Gudrun Bachmann, Odette Haefeli, und Michael Kindt, 87-97. Münster: Waxmann.

Badiee, Farnaz, und David Kaufman. 2014. «Effectiveness of an online simulation for teacher education.» Journal of Technology and Teacher Education 22 (2): 167-86.

Baer, Matthias, Günter Dörr, Urban Fraefel, Mirjam Kocher, Oliver Küster, Susanna Larcher, Peter Müller, Waltraud Sempert, und Corinne Wyss. 2007. «Werden angehende Lehrpersonen durch das Studium kompetenter? Kompetenzaufbau und Standarderreichung in der berufswissenschaftlichen Ausbildung an drei Pädagogischen Hochschulen in der Schweiz und in Deutschland.» Unterrichtswissenschaft 35 (1): 15-47.

Baer-Baldauf, Pascale. 2020. Wie gestalten Hochschulen ein praxisbezogenes Studium? Eine vergleichende Fallstudienuntersuchung von Praxiselementen und förderlichen institutionellen Rahmenbedingungen in Bachelorstudiengängen der Betriebswirtschaftslehre in Deutschland und in der Schweiz. Untersiemau: Difo-Druck. http://verdi.unisg.ch/www/edis.nsf/ SysLkpByIdentifier/4933/\$FILE/dis4933.pdf.

Baran, Evrim. 2014. «A Review of Research on Mobile Learning in Teacher Education.» Educational Technology \& Society 17 (4): 17-32.

Bartel, Marie-Elene, und Jürgen Roth. 2015. «Diagnostische Kompetenz durch Videovignetten fördern.» In Beiträge zum Mathematikunterricht 2015, herausgegeben von Franco, Helmut Linneweber-Lammerskitten, und Christine Streit, 1033-36. Münster: WTM.

Bäuerlein, Kerstin, und Urban Fraefel. 2016. «Ein Video-Portfolio zur summativen Überprüfung berufspraktischer Kompetenzen.» Beiträge zur Lehrerinnen- und Lehrerbildung 34 (2): 212-18.

Baumgartner, Peter, Klaus Himpsl, und Sabine Zauchner. 2009. Einsatz von EPortfolios an (österreichischen) Hochschulen: Zusammenfassung - Teil I des BMWF-Abschlussberichts E-Portfolio an Hochschulen. Krems: Donau Universität Krems.

Beach, Pamela, und Jen McConnel. 2019. «Eye tracking methodology for studying teacher learning: a review of the research.» International Journal of Research \& Method in Education 42 (5): 485-501. https://doi.org/10.1080/174372 7X.2018.1496415.

Berndt, Constanze, Thomas Häcker, und Tobias Leonhard, Hrsg. 2017. Reflexive Lehrerbildung revisited. Traditionen - Zugänge - Perspektiven. Bad Heilbrunn: Klinkhardt.

Billingsley, Glenna, Scott Smith, Shaunna Smith, und Julia Meritt. 2019. «A Systematic Literature Review of Using Immersive Virtual Reality Technology in Teacher Education.» Journal of Interactive Learning Research 30 (1): 65-90. 
Blomberg, Geraldine, Alexander Renkl, Miriam Gamoran Sherin, Hilda Borko, und Tina Seidel. 2013. "Five research-based heuristics for using video in pre-service teacher education.» Journal for Educational Research Online 5 (1): 90-114.

Boos, Maria, Astrid Krämer, und Meike Kricke, Hrsg. 2016. Portfolioarbeit phasenübergreifend gestalten. Konzepte, Ideen und Anregungen aus der LehrerInnenbildung. Münster: Waxmann.

Borges, Simone, Vinicius Durelli, Helena Reis, und Seiji Isotani. 2014. «A Systematic Mapping on Gamification Applied to Education». In SAC ,14: Proceedings of the 29th Annual ACM Symposium on Applied Computing, 216-22. Association for Computing Machinery, New York. https://doi.org/10.1145/2554850.2554956.

Boulton, Helen. 2014. «ePortfolios beyond pre-service teacher education: a new dawn?» European Journal of Teacher Education 37 (3): 374-89. https://doi.org/10 $.1080 / 02619768.2013 .870994$.

Bradley, Elizabeth Gates, und Brittany Kendall. 2014. «A Review of Computer Simulations in Teacher Education.» Journal of Educational Technology Systems 43 (1): 3-12. https://doi.org/10.2190/ET.43.1.b.

Bucheli, Sandra. 2018. «Praxisintegrierte Reflexionsseminare im Studiengang Logopädie. Lehrveranstaltungen in und mit dem Praxisfeld.» Logos 26 (1): 34-39.

Busse, Annette, und Dorit Bosse. 2019. «Peer-Learning vom Studienbeginn bis ins Referendariat -ePortfolio-gestützte Lernszenarien.» Journal für LehrerInnenbildung 19 (3): 66-72.

Caponetto, Ilaria, Jeffrey Earp, und Michela Ott. 2014. «Gamification and Education: a Literature Review.»I In Proceedings of the 8th European Conference on Games-Based Learning - ECGBL 2014. Bd. 1, herausgegeben von Carsten Busch, 50-57. Red Hook: Curran Associates.

Caruso, Carina. 2019. Das Praxissemester von angehenden Lehrkräften. Ein MixedMethods-Ansatz zur Exploration ausgewählter Effekte. Wiesbaden: Springer VS.

Claussen, Jan Torge, Gitte Köllner, Anna-Katharina Poschkamp, Anja SchwedlerDiesener, Michael Besser, und Torben Schmidt. 2020. Die digitale DidaktikWerkstatt: Einsatz digitaler Medien im Lehramtsstudium an der Leuphana Universität Lüneburg. https://www.e-teaching.org/etresources/pdf/erfahrungsbericht_2020_claussen-et-al_die-digitale-didaktik-werkstatt.pdf.

Christen, Andrea, und Martin Hofmann. 2008. «Implementation of E-Portfolio in the First Academic Year at the University of Teacher Education St. Gallen.» International Journal of Emerging Technologies in Learning 3 (1): 1-10.

Christensen, Rhonda R., Gerald Knezek, Tandra Tyler-Wood, und David C. Gibson. 2011. «SimSchool: An online dynamic simulator for enhancing teacher preparation.» International Journal of Learning Technology 6 (2): 201-20. https://doi. org/10.1504/IJLT.2011.042649.

Cortina, Kai S., Katharina Müller, Janina Häusler, Kathleen Stürmer, Tina Seidel, und Kevin F. Miller. 2018. «Feedback mit eigenen Augen: Mobiles Eyetracking in der Lehrerinnen- und Lehrerbildung.» Beiträge zur Lehrerinnen- und Lehrerbildung 36 (2): 208-222. 
Cramer, Cramer, und Lina Feder. 2018. «Potenziale von Portfolioarbeit in der Lehrerbildung.» DDS - Die Deutsche Schule 110 (4): 354-68. https://doi. org/10.31244/dds.2018.04.06.

Cooper, Harris M., Larry V. Hedges. 2009. «Research Synthesis as a Scientific Process.» In The Handbook of Research Synthesis and Meta-Analysis, herausgegeben von Harris M. Cooper, Larry V. Hedges, und Jeffrey C. Valentine, 3-16. New York: Sage.

Cress, Ulrike, Ira Diethelm, Birgit Eickelmann, Olaf Köller, Reinhold Nickolaus, Hans Anand Pant, und Kristina Reiss. 2018. Schule in der digitalen Transformation - Perspektiven der Bildungswissenschaften. München: acatech.

de Witt, C., und C. Gloerfeld, hrsg. 2018. Handbuch Mobile Learning. Wiesbaden: Springer VS.

Deale, Deb, und Ray Pastore. 2014. «Evaluation of simSchool: An Instructional Simulation for Pre-Service Teachers.» Computers in the Schools 31 (3): 197-219. https://doi.org/10.1080/07380569.2014.932650.

Dichev, Christo, und Darina Dicheva. 2017. "Gamifying education: what is known, what is believed and what remains uncertain: a critical review.» International Journal of Educational Technology in Higher Education 14 (9): . https://doi. org/10.1186/s41239-017-0042-5.

Dicheva, Darina, Christo Dichev, Gennady Agre, und Galia Angelova. 2015. «Gamification in Education: A Systematic Mapping Study.» Educational TechnologY \& Society 18 (3): 75-88.

Drossel, Kerstin, Birgit Eickelmann, Heike Schaumburg, und Amelie Labusch. 2019. «Nutzung digitaler Medien und Prädiktoren aus der Perspektive der Lehrerinnen und Lehrer im internationalen Vergleich.» In ICILS 2018 \#Deutschland. Computer- und informationsbezogene Kompetenzen von Schülerinnen und Schülern im zweiten internationalen Vergleich und Kompetenzen im Bereich Computational Thinking, herausgegeben von Birgit Eickelmann, Wilfried Bos, Julia Gerick, Frank Goldhammer, Heike Schaumburg, Knut Schwippert, Martin Senkbeil, und Jan Vahrenhold, 205-40. Münster: Waxmann.

Duchowski, Andrew T. 2002. "A breadth-first survey of eye-tracking applications.» Behavior Research Methods, Instruments, \& Computers 34: 455-70.

Duchowski, Andrew T. 2017. Eye tracking methodology. Theory and practice. Cham: Springer.

Fraefel, Urban, Kerstin Bäuerlein, und Antje Barabasch. 2018. «Assessing teacher candidates' professional competence for evaluating teacher education programs: The case of German-speaking Europe.»In Handbook of Research on Program Development and Assessment Methodologies in K-20 Education, herausgegeben von Victor Wang, 418-42. Hershey: IGI Global.

Friesen, Marita Eva, Jasmin Benz, Tim Billion-Kramer, Christian Heuer, Hendrik Lohse-Bossenz, Mario Resch, und Juliane Rutsch, Hrsg. 2020. Vignettenbasiertes Lernen in der Lehrerbildung. Fachdidaktische und pädagogische Perspektiven. Weinheim: Beltz Juventa. 
Fromme, Johannes, und Dominik Petko, Hrsg. 2008. Computerspiele und Videogames in formellen und informellen Bildungskontexten. MedienPädagogik. Zeitschrift für Theorie und Praxis der Medienbildung, Nr. 15. https://doi.org/10.21240/ mpaed/15_16.X.

Fortenbacher, Albrecht, Manuel Ninaus, Haeseon Yun, René Helbig, und Korbinian Moeller. 2019. "Sensor Based Adaptive Learning - Lessons Learned.»In DELFI 2019. Proceedings, herausgegeben von Niels Pinkwart und Johannes Konert, 193-98. Bonn: Gesellschaft für Informatik. https://doi.org/10.18420/ delfi2019_355.

Fromm, Martin, und Gabriele Strobel-Eisele, Hrsg. 2020. Praxisbetreuung im Lehramtsstudium. Münster: Waxmann.

Gaudin, Cyrille, und Sébastien Chaliès. 2015. «Video viewing in teacher education and professional development: A literature review.» Educational Research Review 16: 41-67. https://doi.org/10.1016/j.edurev.2015.06.001.

Gröschner, Alexander, Susi Klaß, und Mathias Dehne. 2018. ««Praxis digital». Einsatz neuer Medien-Tools im Praxissemester am Beispiel des onlinebasierten Videofeedbacks.» In Praxisphasen in der Lehrerbildung im Fokus der Bildungsforschung, herausgegeben von Martin Rothland und Ina Biederbeck, 197-207. Münster: Waxmann.

Gröschner, Alexander, Susi Klaß, und Iris Winkler. 2019. "Lernbegleitung von Langzeitpraktika - Konzeption und Designelemente einer hochschuldidaktischen Intervention mittels Unterrichtsvideos.» In Langzeitpraktika als Lernräume - Historische Bezüge, Konzeptionen und Forschungsbefunde, herausgegeben von Julia Košinár, Alexander Gröschner, und Ulrike Weyland, 85-101. Münster: Waxmann.

Grubesic, Katharina, Reinhard Bauer, Klaus Himpsl-Gutermann, und Gerhilde Meissl-Egghart. 2018. «Ich sehe was, was du nicht siehst: Videoreflexion im digitalen Raum. Ein Praxisbericht.» In Digitalisierung und Hochschulentwicklung. Proceedings zur 26. Tagung der Gesellschaft für Medien in der Wissenschaft, herausgegeben von Barbara Getto, Patrick Hintze, und Michael Kerres, 222-33. Münster: Waxmann.

Hascher, Tina. 2011. «Vom «Mythos Praktikum» ...und der Gefahr verpasster Lerngelegenheiten.» Journal für Lehrerinnen- und Lehrerbildung 11 (3): 8-16.

Hedtke, Reinhold. 2019. "Wissenschaft und Weltoffenheit. Wider den Unsinn der praxisbor-nierten Lehrerausbildung.»In Wieviel Wissenschaft braucht die Lehrerbildung? Zum Stellenwert von Wissenschaftlichkeit im Lehramtsstudium, herausgegeben von Claudia Scheid und Thomas Wenzl, 79-108. Wiesbaden: Springer VS.

Herbst, Patricio, Daniel Chazan, Vu Minh Chieu, Amanda Milewski, Karl W. Kosko, und Wendy Rose Aaron. 2016. «Technology-Mediated Mathematics Teacher Development: Research on Digital Pedagogies of Practice.» In Handbook of Research on Transforming Mathematics Teacher Education in the Digital Age, herausgegeben von Margaret Niess, Shannon Driskell, und Karen Hollebrands, 78-106. Hershey: IGI Global. http://doi:10.4018/978-1-5225-0120-6.choo4. 
Herbst, Patricio, Talli Nachlieli, und Daniel Chazan. 2011. «Studying the Practical Rationality of Mathematics Teaching: What Goes Into 〈Installing〉 a Theorem in Geometry?» Cognition and Instruction 29 (2): 218-55. https://doi.org/10.1080 /07370008.2011.556833.

Hesse, Florian, und Will Lütgert, Hrsg. 2020. Auf die Lernbegleitung kommt es an! Konzepte und Befunde zu Praxisphasen in der Lehrerbildung. Bad Heilbrunn: Klinkhardt.

Hixon, Emily, und Hyo-Jeong So. 2009. «Technology's Role in Field Experiences for Preservice Teacher Training.» Educational TechnologY \& Society 12 (4): 294304.

Hofmann, Florian, Nicole Wolf, Susi Klaß, Isabelle Grassmé, und Michaela GläserZikuda. 2016. «Portfolios in der LehrerInnenbildung. Ein aktueller Überblick zur empirischen Befundlage.» In Portfolioarbeit phasenübergreifend gestalten - Konzepte, Ideen und Anregungen aus der LehrerInnenbildung, herausgegeben von Maria Boos, Astrid Krämer, und Meike Kricke, 23-39. Münster: Waxmann.

Hummel, Hans, Walter Geerts, Aad Slootmaker, Derek Kuipers, und Wim Westera. 2015. «Collaboration scripts for mastership skills: online game about classroom dilemmas in teacher education.» Interactive Learning Environments 23 (6): 670-82. https://doi.org/10.1080/10494820.2013.789063.

Imlig-Iten, Nina, und Dominik Petko. 2018. «Comparing Serious Games and Educational Simulations: Effects on Enjoyment, Deep Thinking, Interest and Cognitive Learning Gains.» Simulation \& Gaming 49 (4): 401-22. https://doi. org/10.1177/1046878118779088.

Jarodzka, Halszka, Kenneth Holmqvist, und Hans Gruber. 2017. «Eye tracking in Educational Science: Theoretical frameworks and research agendas.» Journal of EYe Movement Research 10 (1): 1-18. https://doi.org/10.16910/jemr.10.1.3.

Karpa, Dietrich, Julian Kempf, und Dorit Bosse. 2013. «Das E-Portfolio in der Lehrerbildung aus Perspektive von Studierenden.» Digitale Medien und Schule 4 (7): 1-14.

Kerres, Michael. 2018. Mediendidaktik. Konzeption und Entwicklung digitaler Lernangebote. Berlin: de Gruyter.

Kersting, Nicole. 2008. «Using Video Clips of Mathematics Classroom Instruction as Item Prompts to Measure Teachers' Knowledge of Teaching Mathematics.» Educational and Psychological Measurement 68 (5): 845-61. https://doi. org/10.1177/0013164407313369.

Kleiner, Konrad, Gergely Rakoczi, und Stefan Krimm. 2012. «Blickbewegungsverläufe (mobiles Eye-Tracking) als Element sportdidaktischer Lehr-Lern-Forschung.»In Fachdidaktik Bewegung und Sport im Kontext: Zwischen Orientierung und Positionierung, herausgegeben von Konrad Kleiner, 138-52. Purkersdorf: Brüder Hollinek.

Kleinknecht, Marc, und Alexander Gröschner. 2016. «Fostering preservice teachers' noticing with structured videofeedback: Results of an online- and video-based intervention study.» Teaching and Teacher Education 59: 45-56. https://doi.org/10.1016/j.tate.2016.05.020. 
Kleinknecht, Marc, und Jürgen Schneider. 2013. «What do teachers think and how do they feel when they analyze videos of themselves teaching and of other teachers teaching?» Teaching and Teacher Education 33: 13-23. https://doi. org/10.1016/j.tate.2013.02.002.

Klingsieck, Katrin B., Daniel Al-Kabbani, Carla Bohndick, Johanna Hilkenmeier, Sebastian T. König, Hanna S. Müsche, Saskia Praetorius, und Sabrina Sommer. 2016. "Spielend eine diagnostisch kompetente Lehrkraft werden - mit der game- und e-learningbasierten, problemorientierten und selbstgesteuerten Lernumgebung GEProS.» die hochschullehre 2. Verfügbar unter www. hochschullehre.org.

KMK, Hrsg. 2017. Bildung in der digitalen Welt. Strategie der Kultusministerkonferenz. Berlin: Sekretariat der Kultusministerkonferenz. https://www.kmk.org/ fileadmin/Dateien/pdf/PresseUndAktuelles/2018/Digitalstrategie_2017_mit_ Weiterbildung.pdf.

König, Claudia M. 2019. «Peervideofeedback. Ein Blended-Learning-Konzept in der ersten Phase der Lehrer*innenbildung.» In Teaching Trends 2018. Die Präsenzhochschule und die digitale Transformation, herausgegeben von Susanne Robra-Bissantz, Oliver J. Bott, Norbert Kleinefeld, Kevin Neu, und Katharina Zickwolf, 113-20. Münster: Waxmann.

Könings, Karen, Jean van Berlo, Richard Koopmans, Henk Hoogland, Ingrid Spanjers, Jeroen ten Haaf, Cees van der Vleuten, und Jeroen van Merrienboer. 2016. «Using a Smartphone App and Coaching Group Sessions to Promote Residents' Reflection in the Workplace.» Academic Medicine 91 (3): 365-70. https:// doi.org/10.1097/ACM.0000000000000989.

Košinár, Julia, Alexander Gröschner, und Ulrike Weyland, Hrsg. 2019. Langzeitpraktika als Lernräume - Historische Bezüge, Konzeptionen und Forschungsbefunde. Münster: Waxmann.

Latoschik, Marc Erich, Florian Kern, Jan-Philipp Stauffert, Andrea Bartl, Mario Botsch, und Lugrin, Jean- Luc. 2019. "Not Alone Here?! Scalability and User Experience of Embodied Ambient Crowds in Distributed Social Virtual Reality.» IEEE Transactions on Visualization and Computer Graphics 25 (5): 2133-44. https://doi.org/10.1109/TVCG.2019.2899250.

Leonhard, Tobias. 2018. «Das Ende von Theorie und Praxis? Versuch einer alternativen Rahmung für die Lehrerinnen- und Lehrerbildung.» In Forschungsperspektiven 10, herausgegeben von Christian Fridrich, Gabriele Mayer-Frühwirth, Renate Potzmann, Wolfgang Greller, und Ruth Petz, 11-26. Münster: LIT.

Lugrin, Jean-Luc, Marc Erich Latoschik, Michael Habel, Daniel Roth, Christian Seufert, und Silke Grafe. 2016. «Breaking Bad Behaviors: A New Tool for Learning Classroom Management Using Virtual Reality.» Frontiers in ICT 3: 26. https://doi.org/10.3389/fict.2016.00026. 
Lugrin, Jean-Luc, Sebastian Oberdörfer, Marc Erich Latoschik, Alice Wittmann, Christian Seufert, und Silke Grafe. 2018. «VR-Assisted vs Video-Assisted Teacher Training.» In 25th IEEE Conference on Virtual Reality and 3D User Interfaces (VR), herausgegeben von Kiyoshi Kiyokawa, Frank Steinicke, Bruce H. Thomas, und Greg Welch, 625-26. Piscataway: IEEE. https://doi.org/10.1109/ VR.2018.8446312.

MacEntee, Virginia, und Barbara Garii. 2010. «E-Portfolios in Teacher Education.» In The E-Portfolio Paradigm: Informing, Educating, Assessing, and Managing With E-Portfolios, herausgegeben von Nicole Buzzetto-More, 191-205. Santa Rosa: Informing Science Press.

Makrinus, Livia. 2013. Der Wunsch nach mehr Praxis. Zur Bedeutung von Praxisphasen im Lehramtsstudium. Wiesbaden: Springer VS.

Mayer-Frühwirth, Gabriele. 2017. «Social Video Learning. Kollaboratives Reflektieren in den Schulpraktika». In Forschungsperspektiven 9, herausgegeben von Christian Fridrich, Gabriele Mayer-Frühwirth, Renate Potzmann, Wolfgang Greller, und Ruth Petz, 69-76. Münster: LIT.

McPherson, Rebekah, Tandra Tyler-Wood, Amber McEnturff Ellison, und Pamela Peak. 2011. «Using a Computerized Classroom Simulation to Prepare PreService Teachers.» Journal of Technology and Teacher Education 19 (1): 93-110.

Meyer, Robert, und Julia Aulinger. 2019. «UnterrichtOnline.org - ein Videografieportal für alle Phasen der LehrerInnenbildung. Interaktives und kollaboratives webbasiertes Lehren und Lernen mit Unterrichtsvideos.» In DELFI 2019. Proceedings, herausgegeben von Niels Pinkwart und Johannes Konert, 331-32. Bonn: Gesellschaft für Informatik. https://doi.org/10.18420/delfi2019_231.

Miller, Damian, und Benno Volk, Hrsg. 2013. E-Portfolio an der Schnittstelle von Studium und Beruf. Münster: Waxmann.

Monitor Lehrerbildung, Hrsg. 2018. Lehramtsstudium in der digitalen Welt - Professionelle Vorbereitung auf den Unterricht mit digitalen Medien?! Gütersloh: CHE Centrum für Hochschulentwicklung. https://www.monitor-lehrerbildung.de/ export/sites/default/.content/Downloads/Monitor-Lehrerbildung_Broschuere_Lehramtsstudium-in-der-digitalen-Welt.pdf.

Nah, Fiona Fui-Hoon, Qing Zeng, Venkata Rajasekhar, Telaprolu, Abhishek, Padmanabhuni Ayyappa, und Brenda Eschenbrenner. 2014. "Gamification of Education: A Review of Literature.》 In First International Conference on HCI in Business. Proceedings, herausgegeben von Nah, Fiona Fui-Hoon, 401-09. Cham: Springer.

Newman, Mark, und David Gough. 2020. «Systematic Reviews in Educational Research: Methodology, Perspectives and Application.» In Systematic Reviews in Educational Research, herausgegeben von Olaf Zawacki-Richter, Michael Kerres, Svenja Bedenlier, Melissa Bond, und Katja Buntins, 3-22. Wiesbaden: Springer VS.

Oelkers, Jürgen. 2000. Studium als Praktikum? Illusionen und Aussichten der Lehrerbildung. https://www.sowi-online.de/journal/2000_o/oelkers_studium_praktikum.html. 
Parker, Michele, Abdou Ndoye, und Albert D. Ritzhaupt. 2012. "Qualitative Analysis of Student Perceptions of E-Portfolios in a Teacher Education Program.» Journal of Digital Learning in Teacher Education 28 (3): 99-107. https://doi.org/1 0.1080/21532974.2012.10784687.

Petko, Dominik, Beat Döbeli Honegger, und Doreen Prasse. 2018. «Digitale Transformation in Bildung und Schule: Facetten, Entwicklungslinien und Herausforderungen für die Lehrerinnen- und Lehrerbildung.» Beiträge zur Lehrerinnen- und Lehrerbildung 36 (2): 157-74.

Petko, Dominik, Doreen Prasse, und Kurt Reusser. 2014. "Online-Plattformen für die Arbeit mit Unterrichtsvideos: Eine Übersicht.» Beiträge zur Lehrerinnenund Lehrerbildung 32 (2): 247-61.

Petko, Dominik, Regina Schmid, Laura Müller, und Michael Hielscher. 2019. «Metapholio: A Mobile App for Supporting Collaborative Note Taking and Reflection in Teacher Education.» TechnologY, Knowledge and Learning 24: 699-710. https://doi.org/10.1007/s10758-019-09398-6.

Piwowar, Valentina, Victoria L. Barth, Diemut Ophardt, und Felicitas Thiel. 2018. «Evidence-based scripted videos on handling student misbehavior: the development and evaluation of video cases for teacher education.» Professional Development in Education 44 (3): 369-84. https://doi.org/10.1080/19415257.201 7.1316299.

Praetorius, Saskia, Daniel Al-Kabbani, Carla Bohndick, Johanna Hilkenmeier, Sebastian T. König, Hanna S. Müsche, Sabrina Sommer, und Katrin B. Klingsieck. 2016. "Spielend Lehrer/in werden: problembasiertes Lernen mit virtuellen Schülerinnen/Schülern.» Zeitschrift für Hochschulentwicklung 11 (3): 231-45.

Rehfeldt, Daniel, David Seibert, Christiane Klempin, Martin Lücke, Michaela Sambanis, und Volkhard Nordmeier. 2018. «Mythos Praxis um jeden Preis? Die Wurzeln und Modellierung des Lehr-Lern-Labors.» die hochschullehre 4. Verfügbar unter: http://www.hochschullehre.org.

Reintjes, Christian, Gabriele Bellenberg, und Grit im Brahm, Hrsg. 2018. Mentoring und Coaching als Beitrag zur Professionalisierung angehender Lehrpersonen. Münster: Waxmann.

Rheinländer, Kathrin, und Daniel Scholl, Hrsg. 2020. Verlängerte Praxisphasen in der Lehrer*innenbildung. Konzeptionelle und empirische Aspekte der Relationierung von Theorie und Praxis. Bad Heilbrunn: Klinkhardt.

Rothland, Martin. 2020. «Legenden der Lehrerbildung. Zur Diskussion einheitsstiftender Vermittlung von 'Theorie' und ,Praxis' im Studium.» Zeitschrift für Pädagogik 66 (2): 270-287.

Rothland, Martin, und Ina Biederbeck, Hrsg. 2018. Praxisphasen in der Lehrerbildung im Fokus der Bildungsforschung. Münster: Waxmann.

Rothland, Martin, und Sarah Katharina Boecker. 2014. «Wider das Imitationslernen in verlängerten Praxisphasen. Potenzial und Bedingungen des Forschenden Lernens im Praxissemester.» DDS - Die Deutsche Schule 106 (4): 386-97. 
Sasaki, Rhonni, Wendy Goff, Andre Dowsett, David Paroissien, Justin Matthies, Celia Di Iorio, Samuel Montey, S., Rowe, und Gemma Puddy. 2020. "The Practicum Experience during Covid-19 - Supporting Pre-Service Teachers Practicum Experience through a Simulated Classroom.» Journal of Technology and Teacher Education 28 (2): 329-39. https://www.learntechlib.org/ primary/p/216244.

Scheidig, Falk. 2017. «Praxisbezüge arrangieren und analysieren. Der «morphologische Kasten» als Planungs- und Reflexionsinstrument des Praxisbezugs in Studium und Lehre.» In Zukunftslabor Lehrentwicklung. Perspektiven auf Hochschuldidaktik und darüber hinaus, herausgegeben von Markus Weil, 135-59. Münster: Waxmann.

Scheidig, Falk. 2020a. «Unterrichtsvideos - neue Szenarien digitaler Praxisbezüge.» Journal für LehrerInnenbildung 20 (1): 28-41. https://doi.org/0.35468/jlbO1-2020_02.

Scheidig, Falk. 2020b. «Zur curricularen Einbettung von Praxisphasen. Kohärenz im Lehramtsstudium aus der Perspektive der Studiengangsentwicklung.»In Verlängerte Praxisphasen in der Lehrer*innenbildung. Konzeptionelle und empirische Aspekte der Relationierung von Theorie und Praxis, herausgegeben von Kathrin Rheinländer und Daniel Scholl, 89-105. Bad Heilbrunn: Klinkhardt.

Scheidig, Falk. Im Erscheinen. «Digitale Formate des Praxisbezugs im Lehramt. Ein Überblick» In Bildung, Schule, Digitalisierung, herausgegeben von Kai Kaspar, Michael Becker-Mrotzek, Sandra Hofhues, Johannes König, und Daniela Schmeinck. Münster: Waxmann.

Scheidig, Falk, und Monika Holmeier. im Erscheinen. «Learning Analytics aus institutioneller Perspektive: ein Orientierungsrahmen für die hochschulische Datennutzung.» In Digitalisierung in Studium und Lehre gemeinsam gestalten. Innovative Formate, Strategien und Netzwerke, herausgegeben von Hochschulforum Digitalisierung. Wiesbaden: Springer VS.

Schmid, Ulrich, Lutz Goertz, Sabine Radomski, Sabrina Thom, und Julia Behrens. 2017. Monitor Digitale Bildung. Die Hochschulen im digitalen Zeitalter. Gütersloh: Bertelsmann Stiftung. https://doi.org/10.11586/2017014.

Schön, Sandra, Martin Ebner, und Martin Schön. 2016. Verschmelzung von digitalen und analogen Lehr- und Lernformaten. Arbeitspapier Nr. 25. Berlin: Hochschulforum Digitalisierung.

Schrittesser, Ilse, Isolde Malmberg, Ruth Mateus-Berr, und Martin Steger, Hrsg. 2014. Zauberformel Praxis? Zu den Möglichkeiten und Grenzen von Praxiserfahrungen in der LehrerInnenbildung. Wien: New Academic Press.

Seidel, Tina, Geraldine Blomberg, und Kathleen Stürmer. 2010. ««Observer» - Validierung eines videobasierten Instruments zur Erfassung der professionellen Wahrnehmung von Unterricht. Projekt OBSERVE.» In Kompetenzmodellierung. Zwischenbilanz des DFG-Schwerpunktprogramms und Perspektiven des Forschungsansatzes, herausgegeben von Eckhard Klieme, Detlev Leutner, und Martina Kenk, 296-306. Weinheim: Beltz. 
Seidel, Tina, und Kathleen Stürmer. 2014. «Modeling the structure of professional vision in pre-service teachers.» American Educational Research Journal 51 (4): 739-71. https://doi.org/10.3102/0002831214531321.

Seidel, Tina, und Felicitas Thiel. 2017. «Standards und Trends der videobasierten Lehr-Lernforschung.»Zeitschrift für Erziehungswissenschaft, 20 (Supplement 1): 1-21. https://doi.org/10.1007/s11618-017-0726-6.

Sherin, Miriam Gamoran, und Elizabeth A. van Es. 2009. "Effects of video club participation on teachers' professional vision.» Journal of Teacher Education 60 (1): 20-37. https://doi.org/10.1177/0022487108328155.

Sonnleitner, Magdalena, Stefan Prock, Astrid Rank, und Petra Kirchhoff, Hrsg. 2018. Video- und Audiografie von Unterricht in der LehrerInnenbildung. Opladen: Barbara Budrich.

Stringer Keefe, Elizabeth. 2020. «Learning to Practice Digitally: Advancing Preservice Teachers' Preparation via Virtual Teaching and Coaching.» Journal of Technology and Teacher Education 28 (2): 223-32. https://www.learntechlib. org/primary/p/216145/.

Strudler, Neal, und Keith Wetzel. 2011. «Electronic Portfolios in Teacher Education.» Journal of Research on Technology in Education 44 (2): 161-73. https://doi. org/10.1080/15391523.2011.10782584.

Sweeney, Joe, Amanda Milewski, und Joel Amidon. 2018. «On-ramps to professional practice: Selecting and implementing digital technologies for virtual field experiences.» Contemporary Issues in Technology and Teacher Education 18 (4): 670-91.

Totter, Alexandra. 2019. «Bloggen im Praktikum: Weblog als Reflexionsmedium zum Umgang mit Belastungen in der berufspraktischen Ausbildung von angehenden Lehrpersonen?» Dissertation, Innsbruck: Universität Innsbruck. https://nbn-resolving.org/urn:nbn:at:at-ubi:1-5109o.

Tremp, Peter. 2018. «Berufsbezug dank Forschendem Lernen? Zur Attraktivität einer hoch-schuldidaktischen Losung.»bwp@ Berufs- und Wirtschaftspädagogik - online 34: 1-14. http://www.bwpat.de/ausgabe34/tremp_bwpat34.pdf.

Ulrich, Immanuel, und Alexander Gröschner, Hrsg. 2020. Praxissemester im Lehramtsstudium in Deutschland - Wirkungen auf Studierende (Edition Zeitschrift für Erziehungswissenschaft, 9). Wiesbaden: Springer VS.

van Ackeren, Isabell, Stefan Aufenanger, Birgit Eickelmann, Steffen Friedrich, Rudolf Kammerl, Julia Knopf, Kerstin Mayrberger, Heike Scheika, Katharina Scheiter, und Mandy Schiefner-Rohs. 2019. «Digitalisierung in der Lehrerbildung. Herausforderungen, Entwicklungsfelder und Förderung von Gesamtkonzepten.» DDS - Die Deutsche Schule 111 (1): 103-19. https://doi.org/10.31244/ dds.2019.01.10.

van den Bogert, Niek, Jan van Bruggen, Danny Kostons, und Wim Jochems. 2014. «First steps into understanding teachers' visual perception of classroom events.» Teaching and Teacher Education 37: 208-16. https://doi.org/10.1016/j. tate.2013.09.001. 
Vohle, Frank. 2013. «Relevanz und Referenz: Zur didaktischen Bedeutung situations-genauer Videokommentare im Hochschulkontext.» In Hochschuldidaktik im Zeichen von Heterogenität und Vielfalt, herausgegeben von Gabi Reinmann, Martin Ebner, und Sandra Schön, 165-81. Norderstedt: Books on Demand.

Weber, Kira Elena, Bernadette Gold, Christopher Neil Prilop, und Marc Kleinknecht. 2018. "Promoting pre-service teachers' professional vision of classroom management during practical school training: Effects of a structured online-and video-based self-reflection and feedback intervention.» Teaching and Teacher Education 76: 39-49. https://doi.org/10.1016/j.tate.2018.08.008.

Wenzl, Thomas, Andreas Wernet, und Imke Kollmer. 2018. Praxisparolen. Dekonstruktionen zum Praxiswunsch von Lehramtsstudierenden. Wiesbaden: Springer VS.

Weyland, Ulrike. 2010. Zur Intentionalität Schulpraktischer Studien im Kontext universitärer Lehrerausbildung. Paderborn: Eusl.

Weyland, Ulrike, und Eveline Wittmann. 2011. Expertise Praxissemester im Rahmen der Lehrerbildung. 1. Phase an hessischen Hochschulen. Frankfurt: Gesellschaft zur Förderung Pädagogischer Forschung.

Weyland, Ulrike, und Eveline Wittmann. 2015. «Langzeitpraktika in der Lehrerausbildung in Deutschland: Stand und Perspektiven.» Journal für LehrerInnenbildung 15 (1): 8-21.

Wiepke, Axel, Eric Richter, Raphael Zender, und Dirk Richter. 2019. «Einsatz von Virtual Reality zum Aufbau von Klassenmanagement-Kompetenzen im Lehramtsstudium.»In DELFI 2019. Proceedings, herausgegeben von Niels Pinkwart und Johannes Konert, 133-44. Bonn: Gesellschaft für Informatik. https://doi. org/10.18420/delfi2019_319.

Winkel, Jens, Maike Busker, Lisa Schüler, Holger Limberg, und Olaf Jäkel, Hrsg. 2020. Forschendes Lernen im Praxissemester an der Europa-Universität Flensburg. Erfahrungen - Empfehlungen - Perspektiven. Flensburg: University Press.

Wolff, Charlotte E., Halszka Jarodzka, und Henny P.A. Boshuizen. 2017. «See and tell: Differences between expert and novice teachers' interpretations of problematic classroom management events.» Teaching and Teacher Education 66: 295-308. https://doi.org/10.1016/j.tate.2017.04.015.

Wouters, Pieter, Christof van Nimwegen, Herre van Oostendorp, und Erik D. van der Spek. 2013. "A metaanalysis of the cognitive and motivational effects of serious games.» Journal of Educational Psychology 105 (2): 249-65. https://doi. org/10.1037/a0031311.

Zheng, Robert Z., und Michael K. Gardner, Hrsg. 2017. Handbook of research on serious games for educational applications. Hershey: IGI Global. 